Article original

\title{
La flore microbienne de laits crus de vache : diversité et influence des conditions de production
}

\author{
Valérie MicheL ${ }^{a *}$, Agnès HauwuY ${ }^{\mathrm{a}}$, Jean-François CHAMBA ${ }^{\mathrm{b}}$ \\ a SUACI-GIS Alpes du Nord, 11 rue métropole, 73000 Chambéry, France \\ b ITFF, Pré Germain, BP30, 74800 La Roche sur Foron, France
}

(Reçu le 15 septembre 2000 ; accepté le 13 février 2001)

\begin{abstract}
Raw cow milk microflora: diversity and influence of conditions of production . The microbial composition of 158 raw cow milk samples taken from 27 farms located in the French Alps area was determined. Microbial analyses included the total mesophilic aerobic bacteria count as well as numeration of some useful cheesemaking microorganisms (lactic acid bacteria, halophilic bacteria, propionic acid bacteria...), spoilage bacteria (Pseudomonas, enterobacteriaceae, Clostridium spp.) and some of the pathogenic bacteria (coagulase positive staphylococci, Escherichia coli). Milk samples were of good overall microbial quality (mean value less than $10000 \mathrm{cfu} \cdot \mathrm{mL}^{-1}$ ) and had different contents of useful cheesemaking microorganisms or spoilage bacteria. Five different groups of milks were then separated according to their total count and to their relative contents in useful cheesemaking and spoilage microorganisms. When incubated at $37^{\circ} \mathrm{C}$ for $24 \mathrm{~h}$ without any starters addition, milks of the different groups did not have the same ability to produce acid and to coagulate. At the farm level, milk suppliers practices were studied. Farmers differed by their way of washing the milking equipment and by their pre-milking and post-milking udder preparation. Combination of these practices influenced the microbial load and composition of the milk produced.
\end{abstract}

microbial composition / raw milk / farmer practice

Résumé - La composition microbiologique de 158 échantillons de laits crus de vache prélevés à la fin de la traite chez 27 producteurs des départements de Savoie et Haute-Savoie a été étudiée. Seize groupes microbiens ont été choisis parmi les groupes d'intérêt technologique (flore acidifiante mésophile, flore halotolérante, bactéries propioniques...), les groupes d'altération (Pseudomonas, entérobactéries, Clostridium spp.) ou certains groupes potentiellement pathogènes (staphylocoques à coagulase positive, Escherichia coli). Si en moyenne les laits analysés renferment très peu de germes (moyenne inférieure à $10000 \mathrm{ufc} \cdot \mathrm{mL}^{-1}$ ), il est possible de distinguer 5 classes de laits qui diffèrent par leur teneur en flore mésophile aérobie revivifiable (de 1200 à $15000 \mathrm{ufc} \cdot \mathrm{mL}^{-1}$ en moyenne) et par leur proportion en flore d'intérêt technologique et en flore d'altération. Les laits des différentes

* Correspondance et tirés-à-part

Tél. : (33) 479707777 ; Fax : (33) 479850779 ; e-mail : vmichel@suacigis.com 
classes ne possèdent pas les mêmes capacités intrinsèques à l'acidification spontanée et à la coagulation. De même, l'aspect des coagulums obtenus varie. Une enquête réalisée chez tous les producteurs lors du prélèvement des laits montre que leurs pratiques diffèrent pour le lavage du matériel de traite et les soins apportés aux mamelles avant et après la traite. Ces pratiques et leur combinaison influencent le niveau des populations microbiennes et la proportion entre les flores d'intérêt technologique et les flores d'altération.

composition microbienne / lait cru / condition de production

\section{INTRODUCTION}

La flore microbienne du lait cru participe de façon importante à l'établissement des caractéristiques organoleptiques des fromages et ce, indépendamment de la présence des ferments. Des travaux de plus en plus nombreux montrent que les fromages au lait cru ont en général des saveurs plus marquées et plus typiques [3, 4, 10, 15, 21]. Très peu d'auteurs ont cherché à caractériser cette flore et surtout à l'étudier dans sa globalité. Seuls les travaux de Desmasures et al. [13] apportent des informations sur les flores des laits crus destinés à la fabrication de Camembert de Normandie. Ils montrent que la composition microbienne des laits, en particulier pour certains groupes bactériens étroitement impliqués dans la fabrication du Camembert, est globalement stable d'année en année $[11,12]$. En revanche, aucune relation n'a pu être établie par ces auteurs entre la composition microbienne de ces laits et leurs conditions de production [11].

Des travaux plus anciens relient la flore des laits avec leurs conditions de production mais ils ne concernent le plus souvent que la flore mésophile aérobie ou certains groupes d'altération [7, 20,23]. Ainsi, il a été montré qu'un lavage soigné des mamelles avant la traite réduisait le nombre de germes mésophiles aérobies, de bactéries psychrotrophes et de bactéries thermorésistantes présents dans le lait [7,23] sans avoir d'effet sur le niveau de bactéries coliformes, comme confirmé par Mc Kinnon et al. [20]. De même, les différentes techniques de lavage du matériel de traite ont des effets plus ou moins importants sur ces quatre catégories de flores [8]. Il est aussi important de préciser que ces résultats concernaient des laits très pollués $\left(10^{5}\right.$ à $\left.10^{8} \mathrm{cfu} \cdot \mathrm{mL}^{-1}\right)$ qui ne semblent plus être les laits produits actuellement.

De plus, la plupart des études disponibles ne s'intéressent qu'à l'effet d'une seule condition de production prise isolément (lavage du matériel de traite ou lavage des mamelles ou post-trempage) sur la flore des laits. Ainsi, à notre connaissance, aucune étude ne met en relation la composition globale de la flore de laits crus (flore d'intérêt technologique et flore indésirable) avec l'ensemble de leurs conditions de productions, c'est-à-dire avec l'ensemble des pratiques des producteurs [11]. L'objectif de cette étude a donc été de mettre en relation la composition microbienne de laits de troupeaux dont les conditions de production variaient avec les pratiques des producteurs, en nous intéressant plus particulièrement aux pratiques concernant le matériel de traite et l'hygiène de la mamelle.

\section{MATÉRIEL ET MÉTHODES}

\subsection{Caractéristiques des exploitations laitières}

Vingt-sept exploitations laitières des départements de Savoie et Haute-Savoie (France) ont été retenues pour cette étude. Elles ont été choisies pour différer par la taille de leur troupeau, par leur matériel de 
Tableau I. Caractéristiques des 27 exploitations laitières ayant participé à l'étude.

Table I. Characteristics of the 27 dairy farms of the study.

\begin{tabular}{llcr}
\hline Caractéristiques des exploitations & & \multicolumn{2}{c}{ Nombre d'exploitations } \\
& & Hiver & Été \\
\hline nombre de vaches laitières & $<30$ & 13 & 11 \\
& 30 à 50 & 7 & 8 \\
& 50 à 140 & 7 & 8 \\
\hline matériel de traite & salle de traite & 8 & 8 \\
& lactoduc en étable & 10 & 13 \\
& pots & 9 & 6 \\
\hline lavage des mamelles avant la traite & absence & 8 & 9 \\
& lavage collectif a & 8 & 9 \\
& lavage individuel & 11 & 9 \\
\hline
\end{tabular}

a : utilisation pour le lavage et /ou l'essuyage des trayons d'une lavette commune à plusieurs vaches.

a : cleaning or drying of cows udder carried out with one collective towel for many cows.

traite ou par leur façon de préparer la mamelle à la traite (Tab. I). Les trois types de matériel de traite les plus courants étaient représentés. Ils se différencient par la localisation des animaux lors de la traite et par le circuit du lait : la salle de traite est une salle séparée du lieu de logement des animaux et équipée d'un circuit fermé pour le lait (lactoduc). Les deux autres systèmes de traite (lactoduc en étable et pots) permettent de traire les vaches dans leur stalle, le circuit du lait étant soit fermé (cas du lactoduc) soit semi-ouvert (cas des pots). Les manières de laver les mamelles distinguent l'absence de lavage, le lavage collectif (utilisation de lavettes communes à plusieurs vaches pour le lavage et/ou l'essuyage de la mamelle) et les lavages individuels (utilisation d'une lavette par vache pour le lavage et l'essuyage des trayons ou utilisation de douchette). L'emploi de produits désinfectants ou non pour laver les mamelles n'a pas été pris en compte.

\subsection{Prélèvements des échantillons de laits}

Dans la demi-heure qui suivait la fin de la traite, un échantillon de lait était prélevé de manière stérile dans les récipients de stockage (réservoirs ou bidons), conservé dans la glace et acheminé directement au laboratoire pour analyses. Le temps maximal entre le prélèvement et l'analyse de l'échantillon ne dépassait pas $3 \mathrm{~h}$. Les laits ont été prélevés trois jours de suite chez un même producteur et pendant deux saisons (hiver et été). Ils correspondaient aux laits d'une seule traite, celle du matin.

\subsection{Analyses microbiologiques}

Dans chaque échantillon de lait, 16 groupes microbiens ont été dénombrés. Les méthodes suivantes ont été utilisées pour :

- la flore mésophile aérobie revivifiable (FMAR) : dénombrement sur milieu Plate Count Agar (PCA; Biokar Diagnostics, Beauvais, France) additionné de $1 \%$ de lait écrémé stérile, incubation $3 \mathrm{j}$ à $30^{\circ} \mathrm{C}$,

- la flore thermorésistante : dénombrement sur milieu PCA + lait après un chauffage de l'échantillon à $63^{\circ} \mathrm{C}$ pendant $30 \mathrm{~min}$, incubation $3 \mathrm{j}$ à $30^{\circ} \mathrm{C}$, 
- la flore aérobie psychrotrophe : dénombrement sur milieu PCA + lait, incubation $10 \mathrm{j}$ à $7{ }^{\circ} \mathrm{C}$,

- la flore acidifiante mésophile (FAM) : dénombrement sur milieu d'Elliker additionné de $0,025 \mathrm{~g} \cdot \mathrm{L}^{-1}$ de pourpre de bromocrésol et de $1 \mathrm{~g} \cdot \mathrm{L}^{-1}$ d'acétate de thallium selon Chamba et al. [5], incubation $3 \mathrm{j}$ à $30^{\circ} \mathrm{C}$,

- les lactobacilles hétérofermentaires facultatifs (LHF) : gélose sélective (ou FH-agar selon Isolini et al. [17]) additionnée de $50 \mathrm{mg} \cdot \mathrm{L}^{-1}$ de vancomycine (Sigma, Saint Quentin Fallavier, France), incubation $3 \mathrm{j}$ à $37{ }^{\circ} \mathrm{C}$ en anaérobiose (système Anaerocult, Merck, Chelles, France),

- la flore halotolérante : dénombrement sur milieu Tryptone Soja Agar (Biokar) additionné de $4,5 \%$ de $\mathrm{NaCl}$, incubation 3 à $5 \mathrm{j}$ à $23{ }^{\circ} \mathrm{C}$,

- les entérocoques: milieu D-Cocossel (Biomérieux, Marcy l'Etoile, France), incubation 24 à $48 \mathrm{~h}$ à $37^{\circ} \mathrm{C}$,

- les bactéries propioniques : gélose Palpropiobac (Standa Industrie, Caen, France), incubation $6 \mathrm{j}$ à $30^{\circ} \mathrm{C}$ en anaérobiose puis $2 \mathrm{j}$ à $23^{\circ} \mathrm{C}$ en aérobiose,

- les levures et moisissures : dénombrement sur gélose à la pomme de terre (milieu PDA, Biokar), additionnée de $0,14 \%$ d'acide tartrique (Sigma), incubation 3 à $5 \mathrm{j}$ à $23{ }^{\circ} \mathrm{C}$,

- les Pseudomonas: gélose Kielwein (Merck) additionnée de 0,025\% de pénicilline-G (PEN-NA, Sigma) et de $0,01 \mathrm{~g} \cdot \mathrm{L}^{-1}$ de pimaricine (Sigma), incubation $3 \mathrm{j}$ à $23{ }^{\circ} \mathrm{C}$,

- les spores de Clostridium: traitement préalable de l'échantillon $15 \mathrm{~min}$ à $75^{\circ} \mathrm{C}$ puis dénombrement sur bouillon Bryant et Burkey (Biokar) comme recommandé par le Cnerna [9], incubation $7 \mathrm{j}$ à $37^{\circ} \mathrm{C}$ en anaérobiose,
- les spores aérobies : traitement préalable de l'échantillon 15 min à $75^{\circ} \mathrm{C}$ puis dénombrement sur PCA additionné de $2 \mathrm{~g} \cdot \mathrm{L}^{-1} \mathrm{~d}$ 'amidon soluble (Merck), incubation $48 \mathrm{~h}$ à $30^{\circ} \mathrm{C}$,

- les entérobactéries : dénombrement sur milieu Violet Red Bile Glucose Agar (Biokar), incubation $24 \mathrm{~h}$ à $30^{\circ} \mathrm{C}$,

- Escherichia coli : dénombrement des E. coli $\beta$-glucuronidase positive sur Rapid E. coli (Sanofi Pasteur, Marnes la Coquette, France), incubation $24 \mathrm{~h}$ à $44^{\circ} \mathrm{C}$ en anaérobiose,

- Staphylococcus à coagulase positive (SCP) : milieu de Baird Parker (Biokar) additionné de fibrinogène de plasma de lapin, incubation 24 à $48 \mathrm{~h}$ à $37^{\circ} \mathrm{C}$.

Tous les milieux étaient ensemencés dans la masse sauf ceux utilisés pour le dénombrement des Pseudomonas et des levures-moisissures.

Les deux genres appartenant aux bactéries potentiellement pathogènes que sont Listeria et Salmonella n'ont pas été recherchés car leur fréquence dans les laits de cette région est très faible voire exceptionnelle. De ce fait, leur éventuelle mise en évidence n'aurait pas été interprétable.

La numération des cellules somatiques des laits a été effectuée par microscopie à épifluorescence (appareil Fossomatic 360, Foss Electric, Hillerod, Danemark).

\subsection{Test de lactofermentation}

Chaque lait a subi un test de lactofermentation qui consistait à incuber stérilement une aliquote de lait à $37^{\circ} \mathrm{C}$ pendant $24 \mathrm{~h}$. Le $\mathrm{pH}$ final du lait était alors mesuré. Son aspect après incubation a été décrit de la façon suivante : liquide $=$ pas de coagulation ; gélatineux = coagulum de type porcelaine, régulier, homogène, sans sérum; caséeux = coagulum plus ou moins contracté, expulsion de sérum verdâtre; floconneux = coagulum en flocons ou en 
grumeaux, expulsion de sérum laiteux ; $d i-$ géré $=$ coagulum plus ou moins digéré avec expulsion d'une grande quantité de sérum.

\subsection{Enquêtes}

Dans chaque exploitation laitière, une enquête et un suivi de la conduite de la traite ont été réalisés pendant l'un des trois jours de prélèvement. Ceux-ci ont été répétés aux deux saisons de l'étude pour prendre en compte tous changements saisonniers des pratiques des producteurs. Le questionnaire de l'enquête portait sur les caractéristiques de l'exploitation, les pratiques des producteurs vis-à-vis du nettoyage du matériel de traite et de la préparation des mamelles à la traite.

\subsection{Analyse des données}

La matrice de corrélations entre les différents groupes microbiens a été réalisée à l'aide du logiciel Statbox (version 2.5, Grimmer Logiciels, Paris, France).

Pour avoir une vision globale des résultats des dénombrements microbiens sur les 158 échantillons de laits en relation avec les pratiques des producteurs, nous avons adopté la démarche suivante. Nous avons tout d'abord constitué des classes de lait présentant une homogénéité de composition pour les groupes microbiens analysés, puis nous avons relié les différences entre les classes à des différences de caractéristiques des exploitations ou de pratiques des producteurs.

Pour cela, les résultats des dénombrements de la majorité des groupes microbiens (10 groupes parmi les 16) ont été classés à l'aide d'une classification des données par nuées dynamiques en utilisant le logiciel Statbox. Une analyse factorielle discriminante (AFD) sous le même logiciel a permis de confirmer la stabilité de cette classification et de reclasser les individus mal classés. Les groupes microbiens non retenus pour construire les classes de lait ont été : la flore mésophile aérobie revivifiable (dans la mesure où l'objectif de cette étude n'était pas d'étudier la teneur en germes totaux des laits) et les germes très peu présents comme les micro-organismes sporulés, les moisissures et $E$. coli. Le groupe potentiellement pathogène des SCP n'a pas non plus été intégré dans cette construction.

La répartition des producteurs en différentes classes a également été réalisée par la méthode des nuées dynamiques. Les variables prises en compte pour élaborer cette classification étaient : le type de matériel de traite, la méthode de lavage du matériel de traite (1 à 2 fois par jour, utilisation fréquente d'acide ou non), la préparation des mamelles à la traite (lavage, élimination des premiers jets), la réalisation du post-trempage.

Le test de répartition des fréquences a été réalisé par test du chi-2 (logiciel Statbox). Les analyses de variance ont été effectuées à l'aide du logiciel SAS (SAS Institute, Inc. Cary, North Carolina, USA).

\section{RÉSULTATS}

\subsection{Composition microbienne des laits analysés}

Les résultats des dénombrements montrent que pour les 16 groupes microbiens analysés, il existe de grandes variations entre tous les laits. Ainsi, à titre d'exemple, le niveau de la FMAR des différents laits varie entre 200 et $120000 \mathrm{ufc} \cdot \mathrm{mL}^{-1}$. Sa valeur moyenne (hiver + été) est de 5800 ufc $\cdot \mathrm{mL}^{-1}$ (Tab. II).

Certains groupes microbiens sont en moyenne très peu présents dans les laits. Il s'agit des micro-organismes sporulés ( $72 \%$ des laits contiennent moins de 180 spores de Clostridium par L), de E. coli et des moisissures dont le niveau moyen est inférieur à $10 \mathrm{ufc} \cdot \mathrm{mL}^{-1}$ et, dans une 
moindre mesure, des entérobactéries, des levures, des bactéries propioniques et des LHF dont le niveau moyen reste inférieur à 50 ufc $\cdot \mathrm{mL}^{-1}$ (Tab. II).

Il est à noter que le niveau moyen de la FMAR est plus faible en hiver (3 700 ufc. $\mathrm{mL}^{-1}$ ) qu'en été (9 $100 \mathrm{ufc} \cdot \mathrm{mL}^{-1}$ ) et reflète les niveaux moyens plus faibles de tous les groupes microbiens en hiver (résultats non montrés).

\subsection{Relations entre les différents groupes microbiens}

Au cours de l'année, il y a globalement très peu de corrélations entre les différents groupes microbiens. Les groupes pour lesquels les coefficients de corrélations sont les plus élevés $(\mathrm{R}>0,55)$ sont : la FMAR et la FAM $(\mathrm{R}=0,78)$, les Pseudomonas et les entérobactéries $(\mathrm{R}=0,62)$, les entérocoques et la flore halotolérante $(\mathrm{R}=0,60)$ et enfin les Pseudomonas et la flore psychrotrophe dans son ensemble $(\mathrm{R}=0,58)$, avec des seuils de signification pour $\mathrm{R}$ tous inférieurs à 0,001 .

\subsection{Représentation et dénomination des classes de lait}

Cinq classes de lait ont été individualisées en classant les résultats des dénombrements microbiens par la méthode des nuées dynamiques. L'AFD a permis de confirmer la stabilité de cette classification : seuls 6 laits sur 158 ont été reclassés.

La figure 1 montre la position des variables et la cartographie des laits sur les deux premiers axes de l'AFD. Le premier axe représente $67 \%$ de la variabilité. Il oppose les laits en fonction de leur teneur croissante pour l'ensemble des groupes microbiens, c'est-à-dire en fonction de leur niveau de FMAR. Il différencie ainsi la classe de lait 1 la plus pauvre (classe P), les classes 2 et 5 (classes M) qui ont des niveaux moyens de flore mésophile aérobie revivifiable inter- médiaires (4 000 et $5400 \mathrm{ufc} \cdot \mathrm{mL}^{-1}$ ), les classes 3 et 4 (classes F) dont les niveaux moyens de FMAR sont plus élevés (13 $\left.800 \mathrm{ufc} \cdot \mathrm{mL}^{-1}\right)$.

Le second axe de l'AFD (23\% de la variabilité) oppose les flores d'intérêt technologique (entérocoques, flore halotolérante, LHF, bactéries propioniques et, dans une moindre mesure, la flore thermophile et la FAM) aux flores d'altération (Pseudomonas, entérobactéries). Il permet ainsi d'opposer les classes 2 et 3 aux classes 4 et 5 . Les classes plus riches en flore d'altération (A) (2 et 3) seront donc désignées ci-dessous MA et FA; les classes les plus riches en flore d'intérêt technologique (IT) seront désignées ci-dessous MIT et FIT. La classe $\mathrm{P}$ (ou 1) quant à elle ne se démarque pas par ses critères.

Le troisième axe de l'AFD (10\% de la variabilité) précise les oppositions entre les classes FA et FIT : la classe FA est plus riche en entérobactéries que la classe FIT, cette dernière étant par contre plus riche en bactéries propioniques et LHF.

\subsection{Composition microbienne des 5 classes de lait}

Le tableau II donne les valeurs moyennes de chaque groupe microbien dans les 5 classes de lait. Il permet aussi de dégager les flores majoritaires du lait cru. Ainsi, parmi toutes les flores et quelles que soient les classes, c'est la flore halotolérante qui est dominante : elle représente selon les classes de 31 à $71 \%$ de la FMAR. Ensuite, on retrouve la FAM qui constitue de 19 à $30 \%$ de la flore mésophile aérobie revivifiable. Enfin, les Pseudomonas occupent une place variable puisque leur pourcentage est compris entre $0,6 \%$ et $15 \%$. Les autres groupes microbiens sont minoritaires et, quelles que soient les classes, ne constituent en moyenne jamais plus de $10 \%$ de la flore mésophile aérobie revivifiable. 
A

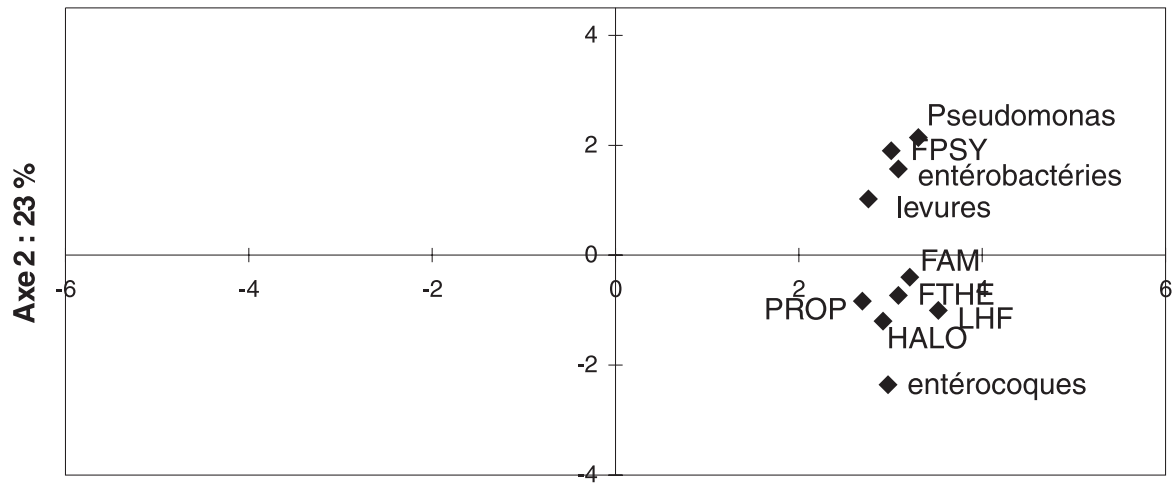

Axe 1: $67 \%$

B

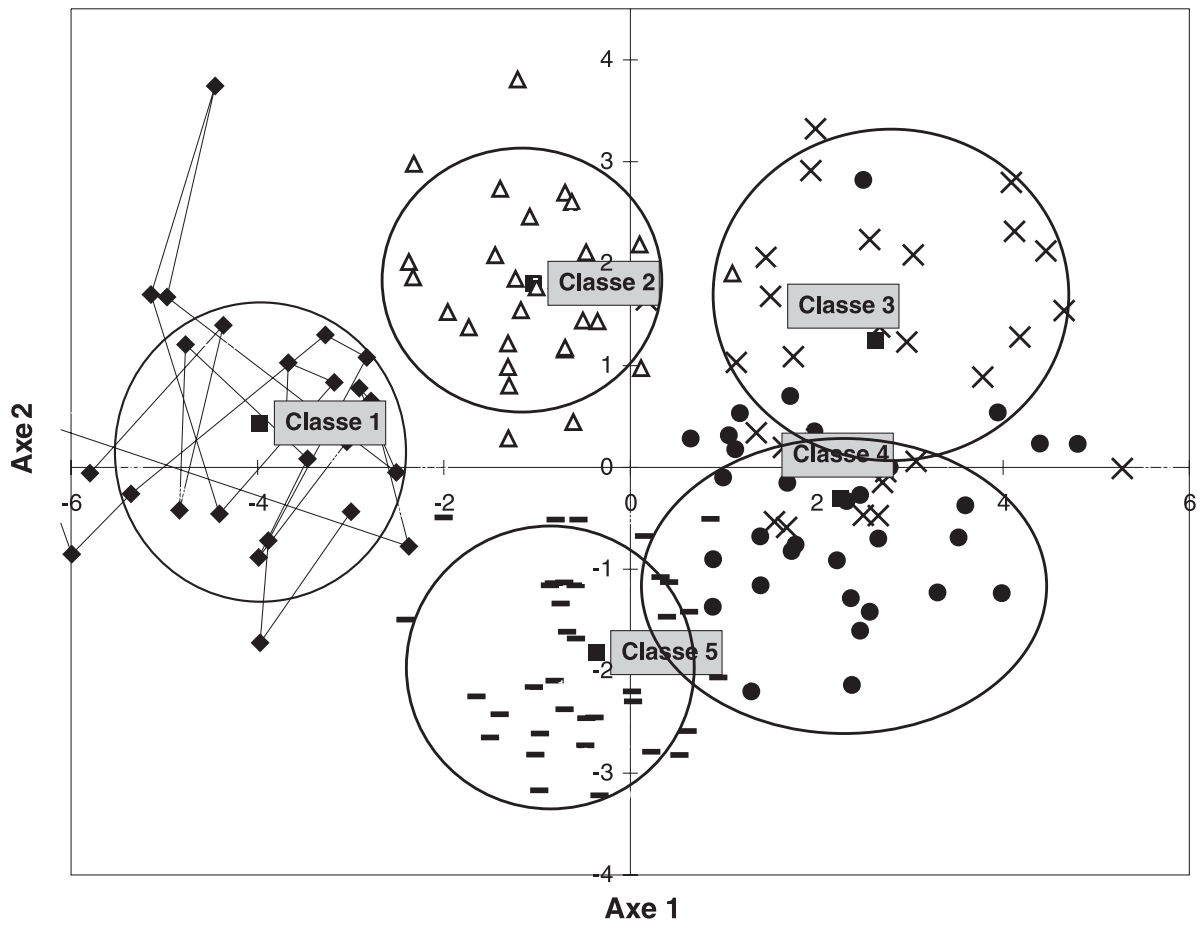

Figure 1. Analyse factorielle discriminante : représentation des variables actives (A), des laits et des classes (B) sur le plan factoriel 1 et 2.

Figure 1. Discriminant factorial analysis: graphic representation of the factors (A), milks and milk groups (B) on the factorial axes 1 and 2. 
Tableau II. Moyennes géométriques des 16 groupes microbiens pour chaque classe de lait. Les niveaux moyens $(\overline{\times})$ et les écarts types $(\sigma)$ des groupes microbiens sont exprimés en $\log \left(\mathrm{ufc} \cdot \mathrm{mL}^{-1}\right)$ sauf pour les spores de Clostridium (log spores $\cdot \mathrm{L}^{-1}$ ). Les classes sont présentées par ordre croissant de flore mésophile aérobie revivifiable totale et nommées selon leur niveau moyen de FMAR $(\mathrm{P}=$ pauvre, $\mathrm{M}=$ moyen, $\mathrm{F}=$ fort) et selon leur richesse en flore d'altération (A) ou en flore d'intérêt technologique (IT).

$\mathrm{FMAR}^{£}=$ flore mésophile aérobie revivifiable, FAM $=$ flore acidifiante mésophile, $\mathrm{LHF}=$ lactobacilles hétérofermentaires facultatifs, $\mathrm{Fl}$. halotolérante $=$ flore halotolérante, Spores $\mathrm{Cl}$. spp. $=$ spores de Clostridium spp., E. coli $=$ Escherichia coli, $\mathrm{SCP}=$ staphylocoques à coagulase positive. a, b, c, d : Les valeurs avec des lettres différentes en exposant sont significativement différentes $(P<0,01)$.

- : flores n'ayant pas servi à la construction des classes.

Analyses de variance sur les log, procédure GLM sous SAS. Signification = ns : non significatif , $*=P<0,05, * * *=P<0,001$.

Table II. Microflora composition of the 5 milk groups.

Results of microbial counts are geometric means $(\overline{\times})$ and standard deviations $(\sigma)$ expressed as log $\left(\mathrm{cfu} \cdot \mathrm{mL}^{-1}\right)$ excepted for Clostridium spores $\left(\log\right.$ spores. $\left.\mathrm{L}^{-1}\right)$. Groups are presented by increasing counts of total mesophilic aerobic flora. They are designated according to their mean FMAR level (low (P), medium (M) or high (F)) and according to the respective amount of alteration bacterial groups (A) or useful cheese-making (IT) microflora.

$\mathrm{FMAR}^{\mathfrak{f}}=$ total mesophilic flora, $\mathrm{FAM}=$ total mesophilic acidifying flora, LHF $=$ facultatively heterofermentative lactobacilli, Fl. halotolérante $=$ halotolerant flora, Spores $\mathrm{Cl}$. spp. $=$ Clostridium spp. spores, E. coli $=$ Escherichia coli, $\mathrm{SCP}=$ coagulase positive staphylococci.

$\mathrm{a}, \mathrm{b}, \mathrm{c}, \mathrm{d}$ : Values with different superscript letters are significantly different $(P<0.01)$.

- : microbial group not used for elaborating the milk groups.

Statistical significance : $\mathrm{ns}=$ not significant, $*=P<0.05, * * *=P<0.001$.

\begin{tabular}{|c|c|c|c|c|c|c|}
\hline Classes & $\mathrm{P}$ & MA & MIT & FIT & FA & Total \\
\hline Effectifs & 26 & 28 & 42 & 32 & 30 & 158 \\
\hline
\end{tabular}

\section{Flores totales}

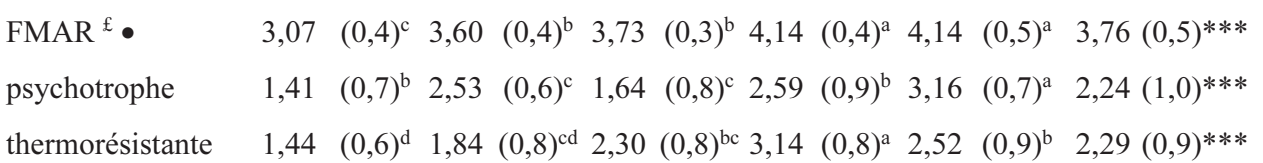

\section{Flores d'intérêt technologique}

\begin{tabular}{|c|c|c|c|c|c|c|c|c|c|c|c|}
\hline$M^{£}$ & 2,44 & $(0,8)^{\mathrm{d}}$ & 2,97 & $(0,4)^{\mathrm{c}}$ & 3,09 & $(0,+7)$ & 3,62 & $(0,5)^{\mathrm{a}}$ & 3 , & $(0,6)^{\mathrm{ab}}$ & 3 , \\
\hline $\mathrm{HF}^{\mathrm{f}}$ & 0,48 & $(0,6)^{\mathrm{d}}$ & 1,26 & $(0,5)^{\mathrm{c}}$ & 1,65 & $(0,7)^{\mathrm{ac}}$ & 2,18 & $(0,6)^{b}$ & 1,72 & $(0,6) \mathrm{a}$ & $1,51(0,8)^{* * *}$ \\
\hline térocoques & 1,22 & $(0,5)^{\mathrm{c}}$ & 1,36 & $(0,7)^{\mathrm{c}}$ & 2,61 & $(0,5)^{b}$ & 2,45 & $(0,4)^{\mathrm{a}}$ & 2,23 & $(0,5)^{\mathrm{ab}}$ & $2,06(0,8)^{* * *}$ \\
\hline Fl. halotolérante ${ }^{£}$ & 2,82 & $(0,4)^{b}$ & 3,09 & $(0,3)^{\mathrm{b}}$ & 3,58 & $(0,5)^{\mathrm{a}}$ & 3,60 & $(0,5)^{\mathrm{a}}$ & 3,63 & $(0,5)^{\mathrm{a}}$ & $3,38(0,5)^{* * *}$ \\
\hline opioniques & 0,84 & $(0,7)^{\mathrm{c}}$ & 1,50 & $(0,8)^{\mathrm{a}}$ & 1,65 & $(0,8)^{\mathrm{a}}$ & 2,39 & $(0,5)^{\mathrm{b}}$ & 1,62 & $(0,7)^{\mathrm{a}}$ & $1,63(0,8)^{* * *}$ \\
\hline vures & 0,54 & $(0,7)^{\mathrm{b}}$ & 1,58 & $(0,9)^{\mathrm{a}}$ & 0,78 & $(0,7)^{\mathrm{b}}$ & 2,07 & $(0,9)^{\mathrm{a}}$ & 1,55 & $(0,9)^{\mathrm{a}}$ & $1,29(1,0)^{* * *}$ \\
\hline oisissures • & 0,54 & $(0,6)$ & 0,73 & $(0,8)$ & 0,71 & $(0,7)$ & 0,96 & $(0,8)$ & 0,99 & $(0,7)$ & $0,79(0,7) \mathrm{ns}$ \\
\hline
\end{tabular}


Tableau II (suite)

Table II (continued).

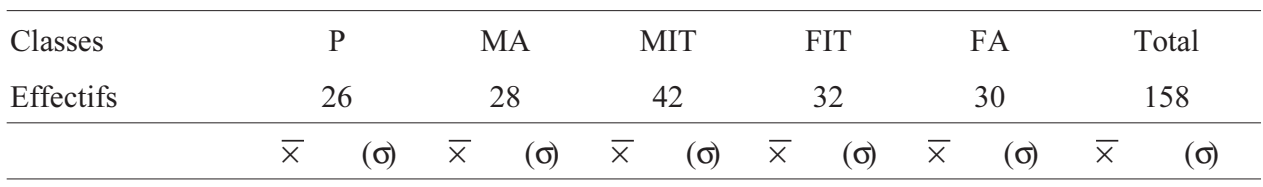

\section{Flores d'altération}

$\begin{array}{lllllllllllll}\text { Pseudomonas } & 1,17 & (1,0)^{\mathrm{c}} & 2,55 & (0,6)^{\mathrm{b}} & 1,48 & (0,8)^{\mathrm{c}} & 2,47 & (0,6)^{\mathrm{b}} & 3,32 & (0,7)^{\mathrm{a}} & 2,17 & (1,1)^{* * *} \\ \text { Entérobactéries } & 0,33 & (0,5)^{\mathrm{c}} & 0,88 & (0,6)^{\mathrm{b}} & 0,63 & (0,7)^{\mathrm{b}} & 0,90 & (0,7)^{\mathrm{bc}} & 2,60 & (0,6)^{\mathrm{a}} & 1,05 & (1,0)^{* * *} \\ \text { Spores } C l . \text { spp. }{ }^{£} \bullet & 0,38 & (0,9) & 1,16 & (1,2) & 0,67 & (1,1) & 0,49 & (0,9) & 0,97 & (1,3) & 0,73 & (1,1)^{*} \\ \text { Spores aérobies } \bullet & 0,35 & (0,4)^{\mathrm{b}} & 0,87 & (0,6)^{\mathrm{a}} & 0,91 & (0,6)^{\mathrm{a}} & 0,81 & (0,8)^{\mathrm{a}} & 0,85 & (0,7)^{\mathrm{a}} & 0,78 & (0,7)^{* *}\end{array}$

Flores potentiellement pathogènes

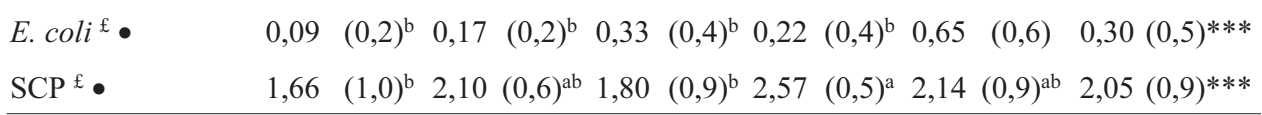

Bien qu'ayant un niveau de FMAR proche des laits de la classe MA, les laits de la classe MIT contiennent plus de flore thermorésistante, de flore halotolérante, d'entérocoques et de levures que les laits de la classe MA. Par contre, les laits de la classe MA renferment plus de Pseudomonas. Les laits des classes FA et FIT, de niveau moyen de FMAR équivalent, ont également des teneurs en flore d'intérêt technologique et flore d'altération différentes : ceux de la classe FA sont les plus riches en flore psychrotrophe, notamment en Pseudomonas, et en entérobactéries. Les laits de la classe FIT contiennent quant à eux davantage de flore thermorésistante, de LHF et de bactéries propioniques.

La répartition des niveaux de certains groupes microbiens dans les différentes classes de lait (résultats non montrés) permet de préciser quelques points. Trente huit pour cent des laits de la classe la plus pauvre en flore totale $(\mathrm{P})$ ont en fait des niveaux de FMAR inférieurs à $1000 \mathrm{ufc} \cdot \mathrm{mL}^{-1}$. Bien qu'ayant un niveau moyen de FMAR très proche, les classes FIT et FA renferment des laits plus ou moins riches en flore acidifiante mésophile. Aucun lait de la classe FIT renferme moins de 1000 germes acidifiants par $\mathrm{mL}$, alors que $30 \%$ des laits de la classe FA ont un niveau de flore acidifiante inférieur à $1000 \mathrm{ufc} \cdot \mathrm{mL}^{-1}$. Les levures sont, elles aussi, présentes en quantité plus importante dans les laits de la classe FIT.

La classe FA est la plus riche en Pseudomonas : $60 \%$ de ses laits en contiennent plus de $1000 \mathrm{ufc} \cdot \mathrm{mL}^{-1}$ alors que pour les classes MA et FIT, les pourcentages correspondants sont de $25 \%$ et $18 \%$ seulement. Quatre-vingt trois pour cent des laits de la classe FA ont un niveau d'entérobactéries dépassant les 100 ufc $\cdot \mathrm{mL}^{-1}$ alors que dans les 4 autres classes, $7 \%$ au maximum des laits dépassent ce seuil. Seules les classes riches en flore d'altération (MA et FA) contiennent des laits pouvant renfermer plus de 500 spores de Clostridium spp. par L (7 et $13 \%$ des laits, respectivement).

Concernant les flores potentiellement pathogènes (non utilisées pour établir la classification), on peut noter qu'il existe pour certaines d'entre elles des différences de niveaux selon les classes. Ainsi, parmi les 5 classes, la classe FA contient le niveau le plus important d'E. coli et la classe FIT 
Tableau III. Répartition des laits de chaque classe en fonction de leur niveau en staphylocoques à coagulase positive.

$\mathrm{SCP}=$ staphylocoques à coagulase positive.

Analyse de répartition des fréquences, test du chi-2 ; différence de répartition entre les classes significatives, $* * *=P<0,001$.

${ }^{£}$ : exprimés en pourcentage des laits de chacune des classes.

Table III. Coagulase-positive staphylococci contents of the five different milk groups.

$\mathrm{SCP}=$ coagulase-positive staphylococci.

Statistical significance: $* * *=P<0.001$.

${ }^{£}$ : expressed as a percentage of the milks of each group.

\begin{tabular}{|c|c|c|c|c|c|c|}
\hline Classes & $\mathrm{P}$ & MA & FA & FIT & MIT & \\
\hline Effectif & 26 & 28 & 30 & 32 & 42 & \\
\hline Niveau de SCP/mL de lait & & & & & & $* * *$ \\
\hline$<100$ ufc & $46^{£}$ & 41 & 37 & 13 & 55 & \\
\hline de 100 à 500 ufc & 46 & 37 & 30 & 50 & 28 & \\
\hline$>500$ ufc & 8 & 22 & 33 & 37 & 17 & \\
\hline
\end{tabular}

contient le niveau moyen le plus important de SCP (Tab. II). Les SCP sont très souvent présents en quantité non négligeable dans les laits : en effet, même dans la classe de niveau moyen de FMAR le plus faible (classe P), plus de la moitié des laits en renferment au moins $100 \mathrm{ufc} \cdot \mathrm{mL}^{-1}$ (Tab. III). Les deux classes de niveau de FMAR le plus élevé (classes FA et FIT) contiennent environ le même pourcentage de laits à plus de $500 \mathrm{SCP}$ par $\mathrm{mL}$ mais se différencient par leur pourcentage de laits contenant moins de $100 \mathrm{SCP}$ par mL (13\% pour la classe FIT contre $37 \%$ pour la classe FA).

\subsection{Niveau de cellules somatiques et acidification spontanée des laits des différentes classes}

Soixante-douze pour cent des laits analysés contenaient moins de 200000 cellules somatiques par $\mathrm{mL}$. Une faible proportion des laits analysés $(8 \%)$ contenait plus de 400000 cellules somatiques par $\mathrm{mL}$, la moitié d'entre eux appartenant à la classe FA. Aucune relation significative n'a été mise en évidence entre le niveau de cellules somatiques dans le lait et son apti- tude à former l'un des 5 types de coagulum décrits précédemment.

Les différences de composition microbienne des 5 classes de laits ont une influence nette sur leur acidification spontanée (Tab. IV). La diminution moyenne de $\mathrm{pH}$ ne reflète pas la capacité de coagulation des laits car, pour une même valeur moyenne de diminution de $\mathrm{pH}$, la proportion de laits qui restent liquides est différente (cas des laits de la classe MA et MIT). Environ 2/3 $(65 \%)$ des laits les plus pauvres en FMAR ne coagulent pas. Pour des niveaux de FMAR plus élevés, on observe une plus forte proportion de laits qui coagulent, cette proportion étant maximale pour les laits de la classe FIT (seuls $3 \%$ restent liquides). Celle-ci est à relier à la forte proportion de flore d'intérêt technologique dans les laits de cette classe. En effet, à même niveau de FMAR, les laits de la classe FA, plus riches en flore d'altération, coagulent moins fréquemment (40\% restent liquides).

Lorsqu'il y a coagulation, le type de coagulum formé dépend du type de flore dominante : ainsi, les laits de la classe la plus riche en flore d'intérêt technologique 
Tableau IV. Acidification spontanée et aspect des coagulums obtenus selon l'appartenance des laits à chacune des 5 classes prédéfinies.

a, b, c : Les valeurs avec des lettres différentes en exposant sont significativement différentes $(P<0,01)$.

Analyse de répartition des fréquences, test du chi-2 ; différence de répartition entre les classes significatives, $* * *=P<0,001$.

$£$ : exprimés en pourcentage des laits de chacune des classes.

Table IV. Acidifying properties and coagulum aspects of the milks belonging to the 5 groups previously described.

Decreasing $\mathrm{pH}$ values were statistically analysed by ANOVA.

a, b, c : Values with different superscript letters are significantly different $(P<0.01)$.

Statistical significance : $* * *=P<0.001$.

${ }^{£}$ : expressed as a percentage of the milks of each group.

\begin{tabular}{lcccccc}
\hline \multicolumn{1}{c}{ Classes } & P & MA & FA & FIT & MIT \\
\multicolumn{1}{c}{ Effectifs } & 26 & 28 & 30 & 32 & 42 & \\
\hline $\begin{array}{l}\text { Diminution moyenne de } \mathrm{pH} \\
\text { Type de coagulum }\end{array}$ & $-1,64^{\mathrm{a}}$ & $-1,76^{\mathrm{ab}}$ & $-1,93^{\mathrm{b}}$ & $-2,24^{\mathrm{c}}$ & $-1,75^{\mathrm{ab}}$ & \\
$\begin{array}{l}\text { liquide } \\
\text { gélatineux }\end{array}$ & $65^{\mathfrak{f}}$ & 37 & 40 & 3 & 50 & \\
caséeux & 19 & 22 & 24 & 69 & 21 \\
floconneux & 8 & 11 & 13 & 6 & 5 & \\
digéré & 8 & 15 & 13 & 9 & 10 \\
\hline
\end{tabular}

(classe FIT) donnent plus fréquemment un coagulum de type gélatineux (69\%) que ceux des autres classes (de 19 à $23 \%$ ). Au contraire, les laits des classes riches en flore d'altération (classes MA et FA) donnent plus fréquemment des coagulums de type caséeux ou floconneux.

\subsection{Constance des laits au sein de chaque classe en fonction des saisons}

En moyenne sur toute la durée de l'étude, pour la majorité des producteurs (54\%), les laits analysés ne voient pas leur classement modifié au cours des trois prélèvements successifs. Ce pourcentage est un peu plus élevé en hiver $(60 \%)$ qu'en été (48\%). Lorsqu'un changement de classe se produit, il ne concerne qu'un échantillon de lait sur trois pour $35 \%$ des producteurs et correspond à un passage dans une classe proche, c'est-à-dire de niveau de FMAR proche (passage de $\mathrm{P}$ à MA ou MIT, de MA à FA ou de FIT à MIT) ou de même niveau de FMAR mais de composition différente (passage de MA à MIT et de FA à FIT). Seuls $11 \%$ des producteurs de cette étude (3 en hiver et en été) ont produit des laits qui appartenaient à 3 classes différentes.

\subsection{Typologie des exploitations laitières}

À chaque producteur étaient associées les variables recueillies le jour de l'enquête. Nous lui avons affecté la classe de lait correspondant au moins aux deux tiers de ses laits. Nous avons ainsi éliminé les 6 exploitations pour lesquelles les 3 laits produits par période appartenaient à 3 classes distinctes.

Les 48 producteurs retenus ont été séparés en 3 classes ou groupes en utilisant la 
Tableau V. Répartition des producteurs dans les différents groupes selon leurs pratiques de traite. a : utilisation peu fréquente d'acide $=$ moins d'une fois tous les 2 jours .

$\mathrm{b}$ : une donnée manquante.

Analyse de répartition des fréquences, test du Chi-2 ; différence de répartition des producteurs entre les trois groupes significatives avec comme seuil : $*=P<0,05$, *** $=P<0,001$.

Table V. Relation between groups of milk farmers and their practices.

${ }^{a}$ : acid used less than once every two days to wash the milking machine.

$\mathrm{b}$ : one missing value.

Statistical significance : difference of repartition of milk producers in between groups is statistically different at $*=P<0.05, * * *=P<0.001$

\begin{tabular}{|c|c|c|c|c|c|}
\hline Groupes de producteurs & I & II & III & Total & \\
\hline Effectif & 18 & 16 & 14 & 48 & \\
\hline Type de matériel de traite & & & & & $* * *$ \\
\hline Lactoduc ou salle de traite, canalisations longueur $15 \mathrm{~m}$ & 14 & & 2 & 16 & \\
\hline Lactoduc ou salle de traite, canalisations longueur $15 \mathrm{~m}$ & 4 & 5 & 10 & 19 & \\
\hline Pots & & 11 & 2 & 13 & \\
\hline Lavage du matériel de traite & & & & & $* * *$ \\
\hline $1 / \mathrm{j}$, acide peu fréquent ${ }^{\mathrm{a}}$ & 14 & 11 & 1 & 26 & \\
\hline $2 / \mathrm{j}$, acide peu fréquent & 1 & 4 & 3 & 8 & \\
\hline $1 / \mathrm{j}$, acide fréquent & 3 & & 6 & 9 & \\
\hline $2 / \mathrm{j}$, acide fréquent & & 1 & 4 & 5 & \\
\hline Lavage des mamelles avant la traite & & & & & $* * *$ \\
\hline pas de lavage & $6^{\mathrm{b}}$ & $11^{\mathrm{b}}$ & & $17^{b}$ & \\
\hline lavage collectif & $7^{\mathrm{b}}$ & $3^{b}$ & 3 & $13^{b}$ & \\
\hline lavage individuel avec ou sans produit & $5^{b}$ & $1^{\mathrm{b}}$ & 11 & $17^{\mathrm{b}}$ & \\
\hline Elimination des premiers jets & & & & & $* * *$ \\
\hline non & 14 & 2 & 2 & 18 & \\
\hline oui & 4 & 14 & 12 & 30 & \\
\hline Post-trempage des mamelles & & & & & $*$ \\
\hline non & 14 & 9 & 4 & 27 & \\
\hline oui & 4 & 7 & 10 & 21 & \\
\hline
\end{tabular}

méthode des nuées dynamiques à partir des critères mentionnés précédemment (pratiques de traite). Les caractéristiques des groupes sont données dans le tableau V.

Les producteurs du groupe I sont équipés en majorité de systèmes de traite dont les longueurs de canalisation sont supérieures à $15 \mathrm{~m}$. Ils lavent leur matériel de traite une fois par jour. Ils se caractérisent par une préparation des mamelles à la traite minimale (absence de lavage des mamelles ou lavage collectif, pas d'élimination des premiers jets) et ne réalisent pas de post-trempage après la traite. Les producteurs du groupe II se distinguent par l'utilisation de systèmes de traite présentant des longueurs 
de canalisation inférieures à 15 mètres et une très forte proportion de producteurs trayant aux pots, l'utilisation peu fréquente (moins d'une fois tous les deux jours) d'acide pour nettoyer le matériel de traite. C'est dans ce groupe que l'on trouve le moins de producteurs qui lavent de façon individuelle les mamelles des vaches. Les producteurs du groupe III diffèrent de ceux du groupe II par leur type de matériel de traite : les producteurs équipés de systèmes de traite aux longueurs de canalisation inférieures à $15 \mathrm{~m}$ sont majoritaires par rapport à ceux équipés de pots. Ils lavent de manière individuelle les mamelles et pratiquent le post-trempage.

Il n'existe pas de lien significatif entre la saison et la répartition des producteurs dans les différents groupes.

\subsection{Association entre les classes de producteurs et les classes de lait}

L'appartenance des laits à chaque classe en fonction de leur conditions de production, c'est-à-dire en fonction des combinaisons de pratiques des producteurs, est donnée dans le tableau VI. Elle montre qu'il existe plusieurs combinaisons de pratiques associées à une même classe de lait (cas des laits des classes FA et MIT). Elle montre également que certaines combinaisons de pratiques sont majoritairement associées ou non à des classes de lait. Ainsi, les producteurs du groupe III produisent des laits appartenant aux classes les plus pauvres et/ou à plus forte proportion de flore d'altération (classes P, MA et FA). Par contre, ils ne produisent jamais de laits appartenant à la classe de niveau le plus élevé en FMAR et à forte proportion de flore d'intérêt technologique (classe FIT). Les producteurs du groupe I ne produisent jamais de laits très pauvres en FMAR (classe P) et une grande majorité d'entre eux produisent des laits appartenant aux classes ayant une forte proportion de flore d'intérêt technologique (classes MIT et FIT). Quant aux producteurs du groupe II, ils ne produisent aucun type de lait préférentiellement.

\section{DISCUSSION}

Les résultats de cette étude montrent la bonne qualité microbiologique globale des

Tableau VI. Relations entre la typologie des producteurs et celle des classes de lait.

Analyse de répartition des fréquences, test du Chi- 2 ; différence de répartition des producteurs entre les trois groupes significative avec comme seuil : $* * *=P<0,001$.

Table VI. Relation between groups of milk farmers and groups of milk.

Statistical significance: difference of repartition of milk producers in between groups is statistically different at $* * *=P<0.001$.

\begin{tabular}{lccccc}
\hline Groupes de producteurs & I & II & III & \multicolumn{2}{c}{ Total } \\
Effectif & 18 & 16 & 14 & 48 & $* * *$ \\
\hline Classes de lait & & & & & 8 \\
P & & 4 & 4 & 8 \\
MA & 1 & 3 & 4 & 9 \\
FA & 2 & 3 & 4 & 11 \\
FIT & 9 & 2 & & 12 \\
MIT & 6 & 4 & 2 & \\
\hline
\end{tabular}


laits de fin de traite produits dans les Alpes du Nord: les laits ont une population moyenne en flore mésophile aérobie revivifiable de 5800 ufc. $\mathrm{mL}^{-1}$ sur toute la durée de l'étude. Bien que ces valeurs ne concernent pas des laits de mélange (laits d'une seule traite, sans étape de conservation), elles sont du même ordre de grandeur que celles obtenues pour des laits crus de mélange prélevés en Normandie [13], au Danemark [1] ou dans des fermes américaines [16].

Ces laits diffèrent par leur richesse respective en flore mésophile aérobie revivifiable. Nous les avons également distingués par leur teneur en flore susceptible de présenter un intérêt technologique ou en flore d'altération.

La répartition quelque peu schématique des groupes microbiens dénombrés entre ces deux types de flore peut être discutée pour certains d'entre eux. Le statut de la flore acidifiante mésophile, des lactobacilles hétérofermentaires facultatifs, des bactéries propioniques, de la flore psychrotrophe et des Pseudomonas ou des Clostridia est évident. En revanche, il l'est moins pour d'autres groupes bactériens. Nous avons considéré que la flore halotolérante telle que nous l'avons dénombrée est essentiellement constituée de bactéries corynéformes (Micrococcus, Arthrobacter, Microbacterium, Brevibacterium...), de staphylocoques banals et de Moraxella qui sont les hôtes habituels de la croûte des fromages [18]. De même, les propriétés des entérocoques, en particulier leur capacité acidifiante sur une large gamme de température, leur permettent de participer à l'élaboration de nombreux fromages traditionnels [26]. D'ailleurs, ils peuvent constituer jusqu'à $10 \%$ de la FMAR [5]. Enfin, les levures et les moisissures participent à l'écosystème microbien de nombreux fromages même si parmi celles-ci on peut trouver aussi bien des espèces d'intérêt technologique que des espèces d'altération. Ce statut peut d'ailleurs varier selon le fromage concerné. Ainsi, Mucor est la moisissure normale de la Tomme de Savoie alors qu'elle est indésirable sur la croûte de Reblochon et de nombreux fromages à pâte molle et à croûte fleurie.

Parmi les flores dominantes de la FMAR, la flore acidifiante mésophile est celle dont la proportion varie le moins. Elle représente en moyenne de $19 \%$ à $30 \%$ de la FMAR selon les classes. Ces résultats sont du même ordre de grandeur que ceux obtenus sur des laits nettement plus peuplés $[6,25]$. Les niveaux de la FMAR et de la FAM sont corrélés. Ainsi, toute action visant à modifier le niveau de FMAR se répercuterait sur le niveau de la FAM, c'est-àdire sur l'un des groupes microbiens d'intérêt technologique qui constitue une proportion non négligeable de la flore mésophile aérobie revivifiable. Les Pseudomonas constituent de $0,6 \%$ à $15 \%$ des laits refroidis et non conservés. Ainsi, leur population varie fortement (d'un facteur 1 à 25) sans que toutefois leur niveau puisse atteindre celui de la FMAR comme dans certains laits de niveau de flore totale équivalent mais conservés 1 à $2 \mathrm{j}$ [13].

L'acidification spontanée des différents laits est liée aux proportions relatives de flore d'intérêt technologique et de flore d'altération des laits. Elle résulte du niveau et de l'équilibre entre la flore d'intérêt technologique et la flore d'altération. La nature du coagulum obtenu varie aussi selon la composition de la flore et ce sont les laits des classes les plus riches en flore d'intérêt technologique qui donnent les caillés les plus structurés (caillés de type gélatineux et caséeux) [19].

Tous les producteurs ne produisent pas le même type de lait. Néanmoins, pour la majorité d'entre eux, les laits produits sont de nature constante (appartenance à une même classe de lait). Ceci laisse présumer que le niveau et la composition des laits ne résultent pas du hasard mais qu'ils dépendent très probablement de leurs conditions de production, c'est-à-dire des pratiques des producteurs. Ainsi, il paraît possible 
d'agir par les conditions de production sur le niveau mais aussi sur la composition de la flore du lait. D'ailleurs, l'association entre les pratiques des producteurs et les classes de lait souligne l'importance du lavage du matériel de traite et des soins apportés aux mamelles d'une part, sur le niveau de flore mésophile aérobie revivifiable et, d'autre part, sur la nature de la flore. Ainsi, le fait que les producteurs du groupe III produisent plus souvent du lait appartenant aux classes de niveau de FMAR le plus réduit peut être expliqué par la forte fréquence du lavage du matériel de traite et des soins apportés aux mamelles. Ces résultats confirment ceux de Chatelin et Richard [7] qui montraient que si le matériel de traite peut être une source importante de micro-organismes, celle-ci est influencée par la technique de lavage du matériel de traite, l'utilisation d'eau chaude acidifiée pour laver le matériel de traite étant l'une des techniques qui réduit le plus l'apport de flore microbienne par le matériel de traite [8]. C'est bien ce que nous observons dans notre étude avec les producteurs du groupe III. A l'inverse, les producteurs du groupe I qui lavent moins intensément leur matériel de traite ne produisent jamais de lait au niveau de FMAR le plus faible. Pour ces producteurs, le fait qu'ils soient surtout équipés de systèmes avec de grandes longueurs de canalisations peut être un élément explicatif supplémentaire. En effet, une installation comprenant de grandes longueurs de canalisation a plus de chances d'apporter un grand nombre de germes dans le lait car la surface de contact et de contamination augmente et que, de plus, on peut trouver plus de zones complexes qui peuvent constituer autant de niches pour le développement des micro-organismes [22], surtout si ce matériel est moins intensément lavé. Toutefois, les producteurs du groupe I produisent des laits contenant une faible proportion de flore d'altération. La peau des mamelles peut être également une source non négligeable de microorganismes [7, 23]. Ainsi, les producteurs du groupe I qui ne lavent pas ou lavent de façon collective les mamelles des vaches avant la traite, ne produisent jamais de laits les plus pauvres en FMAR, corroborant les résultats obtenus par McKinnon et al. [20]. Le lavage des mamelles n'est pas le seul facteur explicatif du niveau de FMAR. En effet, les pratiques de lavage des mamelles avant la traite discriminent peu les producteurs des groupes I et II. Par contre, les producteurs du groupe II se différencient par le fait qu'ils éliminent beaucoup plus les premiers jets avant de poser les faisceaux trayeurs. Les premiers jets récoltés lors de la traite renferment un nombre de germes plus important que le reste du lait [2]. On peut supposer que cette pratique agit comme une purge de l'intérieur du trayon et élimine une forte quantité de micro-organismes. Les producteurs des groupes I et II se différencient également par la réalisation de la technique du post-trempage, celle-ci étant moins utilisée chez les producteurs du groupe I. Cette technique est une désinfection du trayon qui vise à en diminuer les risques d'infection par les germes pathogènes mineurs et majeurs [27]. Nos résultats indiquent que ce type de traitement pourrait avoir un effet plus général sur tous les germes présents en surface ou à l'intérieur des trayons, entraînant ainsi une diminution de la FMAR et de la flore d'intérêt technologique.

Hormis l'influence sur le niveau de FMAR, les pratiques ont des tendances sur la composition de la flore. Ainsi, les pratiques qui préservent la flore des mamelles (absence de lavage des mamelles ou lavage collectif, pas de post-trempage, pas d'élimination des premiers jets) sont plutôt associées à la production de laits riches en flore d'intérêt technologique : les producteurs du groupe I produisent surtout des laits des classes MIT et FIT. La flore d'intérêt technologique telle que nous l'avons définie est constituée en grande partie de flore halotolérante probablement présente dans le sinus du trayon ou en surface des mamel- 
les. Certaines bactéries lactiques peuvent également être hébergées à la surface des mamelles [14] ou, plus généralement, sur le corps des vaches [28]. Une hygiène minimale autour de la mamelle préserverait ce type de flore et permettrait ainsi qu'elle représente une part non négligeable de la FMAR. Cependant ces pratiques se traduiraient très probablement par une contamination en Clostridia du groupe butyrique dans le cas d'une alimentation des vaches laitières à base de fourrages ensilés [24]. Cet affouragement n'étant pas utilisé dans les exploitations étudiées, cela explique les faibles teneurs des laits en spores de Clostridia fermentant le lactate.

La production de laits dont la FMAR est riche en flore d'altération est plus fréquente chez les producteurs appliquant les techniques les plus intenses de lavage du matériel de traite et apportant le plus de soins aux mamelles (lavage individuel et réalisation du post-trempage). On peut probablement mettre en relation cette constatation avec les résultats de Chatelin et Richard [8] selon lesquels l'alternance journalière de produits détergents alcalins et acides conduisait à une plus grande irrégularité du pouvoir contaminant du matériel de traite, la flore microbienne apportée par ce matériel de traite étant alors plus riche en flore d'altération (bactéries coliformes et Pseudomonas). Les producteurs de cette étude dont les pratiques de lavage du matériel de traite se rapprochent le plus de cette technique d'alternance journalière sont le plus représentés dans le groupe III. Or, c'est dans ce groupe que l'on trouve le plus de producteurs produisant des laits à forte proportion de flore d'altération (laits de la classe MA et FA).

Nous pouvons constater dans la composition des laits et dans les pratiques qui leur sont associées une assez nette opposition entre flore d'intérêt technologique et flore d'altération (Pseudomonas, coliformes, Clostridium). Il en est de même pour l'une des deux flores potentiellement pathogènes que nous avons étudiées, $E$. coli qui appartient au groupe des coliformes. Par contre, pour les staphylocoques à coagulase positive qui dans les laits sont représentés en quasi-totalité par Staphylococcus aureus, la situation est moins tranchée. En effet, la classe la plus riche en flore d'intérêt technologique (classe FIT) est aussi la classe qui contient en moyenne le plus de SCP. Pour autant, elle contient environ le même pourcentage de laits à fort niveau de SCP $\left(>500\right.$ ufc $\cdot \mathrm{mL}^{-1}$ ) que la classe FA. Ainsi, si la production de laits appartenant aux classes FIT ou FA est à associer à des combinaisons de pratiques différentes, les combinaisons décrites semblent peu influer sur les forts niveaux de SCP. Pour cela, d'autres éléments sont à prendre en compte au niveau des exploitations comme par exemple, le suivi régulier de l'état sanitaire du troupeau et en particulier, l'éviction des vaches à mammites récurrentes. Les faibles teneurs moyennes en SCP (inférieures à $100 \mathrm{ufc} \cdot \mathrm{mL}^{-1}$ ) observées dans les laits de la classe MIT montre qu'il est possible de produire du lait contenant une forte proportion de flore d'intérêt technologique avec un faible niveau de staphylocoques à coagulase positive.

En conclusion, nous avons donc montré que, malgré leur faible niveau de flore totale, les laits présentaient une diversité de composition microbienne notamment dans la proportion entre les flores d'intérêt technologique et les flores d'altération. Nous avons aussi montré que l'on pouvait associer le niveau de flore des laits et sa composition, non pas à une pratique isolée des producteurs mais bien à une combinaison d'un ensemble de pratiques.

Cependant, pour établir ces associations, d'autres facteurs doivent être pris en compte. En effet, une même combinaison de pratiques conduit à des laits appartenant à différentes classes. Il faudrait peut-être tenir compte d'autres facteurs comme, par exemple, l'état général de propreté de l'exploitation, du lieu de traite et des animaux. 
Il faut certainement améliorer les critères pris en compte pour la description des pratiques en mesurant leur degré d'application ou d'efficacité. Ces informations permettraient alors d'estimer leur impact respectif sur la flore des laits et ainsi d'orienter les pratiques des producteurs vers la production de laits riches en flore d'intérêt technologique tout en limitant la présence des flores d'altération et des flores potentiellement pathogènes. Ainsi, on pourra maintenir le rôle de cette flore dans l'établissement des caractéristiques organoleptiques des fromages au lait cru.

\section{REMERCIEMENTS}

Cette étude a été menée dans le cadre du programme de Recherche \& Développement du GIS Alpes du Nord. Les auteurs remercient tout particulièrement Alexandre Lamarche pour la réalisation des prélèvements et des enquêtes en exploitation. La Fédération Départementale des Coopératives Laitières de Haute-Savoie (FDCL74) est également remerciée pour le financement du premier auteur.

\section{RÉFÉRENCES}

[1] Aagaard K., Jepsen L., Andersen H., Raw milk quality in Denmark, Scand. Dairy Inform. 3 (1998) 22-24

[2] Bacic B., Jackson H., Clegg L., Distribution of bacteria in milk drawn directly from the cow's udder, J. Dairy Sci. 51 (1968) 47-49.

[3] Bouton Y., Grappin R., Comparaison de la qualité de fromages à pâte pressée cuite fabriqués à partir de lait cru ou microfiltré, Lait 75(1995)31-44.

[4] Buchin S., Delague V., Duboz G., Berdagué J.L., Beuvier E., Pochet S., Grappin R., Influence of pasteurization and fat composition of milk on the volatile compounds and flavor characteristics of a semi-hard cheese, J. Dairy Sci. 81 (1998) 3097-3108.

[5] Chamba J.F., Bonnaz G., Bourg P., Efficacité de diverses méthodes de dénombrement de la flore acidifiante du lait cru, Lait 61 (1981) 555-567.

[6] Chamba J.F., Bonnaz G., André P., Fournier J.F. Évolution de la flore microbienne du lait de quatre traites successives au cours du stockage à la ferme en résevoir réfrigérant, Rev. ENIL 61 (1981) $7-15$.

[7] Chatelin Y., Richard J., Étude de quelques cas de contaminations microbiennes importantes du lait à la ferme, Lait 61 (1981) 80-94.

[8] Chatelin Y., Richard J., Comparaison, dans des conditions courantes, de l'efficacité de quatre méthodes de nettoyage des machines à traire, Lait 63 (1983) 87-101.

[9] Cnerna, Recommandations pour l'estimation de la contamination du lait en spores de Clostridia par la méthode de culture en milieu liquide, Rev. Lait Fr. 451 (1986) 39-45.

[10] Demarigny Y., Beuvier E., Buchin S., Pochet S., Grappin R., Influence of raw milk microflora on the characteristics of Swiss-type cheeses: II. Biochemical and sensory characteristics, Lait 77 (1997) 151-167.

[11] Desmasures N., Étude des laits de haute qualité : caractéristiques et aptitude microbiologique à la transformation en camembert au lait cru. Thèse de doctorat de l'université de Caen, 1995.

[12] Desmasures N., Guéguen M., Monitoring the microbiology of high quality milk by monthly sampling over two years, J. Dairy Res. 64 (1997) 271-280.

[13] Desmasures N., Bazin F., Guéguen M., Micro biological composition of raw milk from selected farms in the Camembert region of Normandy, J. Appl. Microbiol. 83 (1997) 53-58.

[14] Desmasures N., Opportune W., Guéguen M., Lactococcus spp., yeasts and Pseudomonas spp. on teats and udders of milking cows as potential sources of milk contamination, Int. Dairy J. 7 (1997) 643-646.

[15] Ginzinger W., Jaros D., Lavanchy P., Rohm H., Raw milk flora affects composition and quality of Bergkäse. 3. Physical and sensory properties, and conclusions, Lait 79 (1999) 411-421.

[16] Hogan J.S., Hoblet K.H., Smith K.L., Todhunter D.A., Schoenberger P.S., Hueston W.D., Pritchard D.E., Bowman G.L., Heider L.E., Brockett B.L., Conrad H.R., Bacterial and somatic cell counts in bulk tank milk from nine well managed herds, J. Food Prot. 51 (1988) 930-934.

[17] Isolini D., Grand M., Glättli H., Selektivmedien zum Nachweis von obligat und fakultativ heterofermentativen Laktobazillen, Schweiz. Milchw. Forschung. 19 (1990) 57-59.

[18] Lefresne G., Irlinger F., Revue taxonomique des bactéries corynéformes, Bull. Soc. Fr. Microbiol. 13 HS (1998) 29-39.

[19] Luquet F., La lactofermentation, Rev. ENIL 6 (1977) 25-32.

[20] McKinnon C., Fulford R., Cousins C., Effect of teat washing on the bacteriological contamination of milk from cows kept under various housing conditions, J. Dairy Res. 50(1983) 153-162. 
[21] McSweeney P.L.H., Fox P.F., Lucey J.A., Jordan K.N., Cogan T.M., Contribution of the indigenuous microflora to the maturation of Cheddar cheese, Int. Dairy J. 3 (1993) 613-634.

[22] Piton C., Efficacité de quelques méthodes de nettoyage des installations de traite dans les conditions courantes des fermes françaises : aspects méthodologiques et résultats pratiques, Tech. Lait. 990 (1984) 39-47.

[23] Piton C., Richard J., Causes de contamination microbienne d'importance moyenne du lait dans un groupe de fermes de la région de Rennes, Lait 62 (1982) 67-74

[24] Rasmussen M., Effects of premilking teat udder preparation on spores of anaerobes, bacteria, and iodine residues in milk, J. Dairy Sci 74 (1991) 2472-2478.
[25] Richard J., Nature de la flore microbienne dominante et sous-dominante des laits crus très pollués, Lait 63 (1983) 148-170.

[26] Richard J., Les entérocoques dans les fromages : une menace discutable pour quelques consommateurs à risque, une amélioration possible de la qualité des fromages au lait cru... ou pasteurisé, Sci. Aliments 20 (2000) 143-152.

[27] Sérieys F., Efficacité des spécialités de pré- et post-trempage des trayons : les essais de terrain, Bull. GTV 3 (1996) 7-18.

[28] Salama M.S., Masafija-Jeknic T., Sandine W.E., Giovannoni S.J., An ecological study of lactic acid bacteria : isolation of new strains of Lactococcus including Lactococcus lactis subsp. cremoris, J. Dairy Sci. 78 (1995) 1004-1017. 